\title{
Lower limb in sprinters with larger relative mass but not larger normalized moment of inertia: novel insights into human plasticity
}

Natsuki Sado ${ }^{1}$ 2 $^{\star}$, Hoshizora Ichinose ${ }^{3}$, Yasuo Kawakami ${ }^{2}$

${ }^{1}$ Faculty of Health and Sports Science, University of Tsukuba, Tsukuba, Japan

2 Faculty of Sports Sciences, Waseda University, Tokorozawa, Japan

${ }^{3}$ Graduate School of Sport Sciences, Waseda University, Tokorozawa, Japan

Corresponding author: Natsuki Sado

E-mail: sado.natsuki.gm@u.tsukuba.ac.jp

ORCiD:

Natsuki Sado: 0000-0002-3958-8193

Yasuo Kawakami: 0000-0003-0588-4039

Author Contributions:

N.S. and Y.K. designed research; N.S. and H.I. performed research; N.S. analyzed data; N.S., H.I., and Y.K. wrote the paper.

Conflict of interest statement:

There are no conflicts of interest to declare.

Classification:

BIOLOGICAL SCIENCES, Physiology

\section{Funding:}

Yamaha motor foundation for sports to N.S. and JSPS KAKENHI Grant-in-Aid for Young Scientists (21K17592) to N.S.

\section{Keywords:}

Biomechanics; Morphology; Adaptation; MRI; Dixon;

\section{This PDF file includes:}

Main Text,

Figures 1 to 3 


\begin{abstract}
Inhomogeneous muscle hypertrophy in response to daily motor executions is a morphological adaptation in humans. Inhomogeneous morphological adaptation would change the mass distribution, which in turn may affect the mechanical difficulty in moving from an inertia perspective; however, the morphological adaptation from the inertia perspective has not been examined. Here, we show no corresponding differences in the normalized mass and normalized moment of inertia between the sprinters and the untrained non-sprinters. We analyzed the fat- and water-separated magnetic resonance images from the lower limbs of 11 male sprinters (100 m best time of 10.44-10.83 s) and 12 untrained non-sprinters. The lowerlimb relative mass was significantly larger in sprinters $(18.7 \pm 0.7 \%$ body mass $)$ than in nonsprinters $(17.6 \pm 0.6 \%$ body mass), while the normalized moment of inertia of the lower limb around the hip in the anatomical position was similar between them $(0.044 \pm 0.002$ vs. $0.042 \pm 0.002$ [a. u.]). The thigh relative mass in sprinters $(12.9 \pm 0.4 \%$ body mass) was significantly larger than that in non-sprinters $(11.9 \pm 0.4 \%$ body-mass $)$, whereas the shank and foot relative masses were similar. We revealed that the adaptation to sprinting in humans does not relatively increase the mechanical difficulty to swing the lower limb in terms of inertia, even though the lower-limb mass increases, reflecting muscular hypertrophy. This is suggested to be one of the significant advantages of human morphology for adaptability to explosive motor tasks.
\end{abstract}

\title{
Significance statement
}

The lower-limb relative mass is larger in sprinters than in non-sprinters, while the normalized moment of inertia of the lower limb around the hip in the anatomical position is similar between them. The present study reveals that the adaptation to sprinting in humans does not relatively increase the mechanical difficulty to swing the lower limb in terms of inertia, even though the lower-limb mass increases, reflecting muscular hypertrophy. These findings suggest a novel significant advantage of adaptability to explosive motor tasks in human morphology. 


\section{Introduction}

Human morphological characteristics are mainly the result of adaptation to bipedal locomotion $(1,2)$. Human morphology enables bipedal walking and endurance running efficiency, resulting in current humans being good endurance runners but poor sprinters (2). Alternately, evolution in humans has left a great plasticity, allowing them to become faster sprinters via training; for example, the Japan National Record of $50 \mathrm{~m}$ sprint is $5.75 \mathrm{~s}$ while the average time of $50-\mathrm{m}$ sprint in Japanese males is approximately $7.37 \pm 0.52 \mathrm{~s}(3)$.

Adaptability in humans has been approached from the muscle morphologies in athletes, based on the exertion abilities of the joint torque (4) and power (5) is primarily determined by the muscle size, especially muscle volume $(4,6)$. Previous MRI studies on sprinters have demonstrated hypertrophy of the trunk and lower-limb muscles (7-9). The hypertrophy was shown to be inhomogeneous with the specific development of the hip flexors and extensors (7-9). The muscle sizes of the hip extensors and flexors are related to the running velocity and/or $100 \mathrm{~m}$ sprint time in sub-elite $(7,8,10)$ and elite sprinters (9). It has also been reported that the hip extensors' volumes discriminate elite sprinters from the subelite (9). The muscularity in sprinters reflects the musculoskeletal demands that increasing running velocity in the high-speed range $(>7 \mathrm{~m} / \mathrm{s})$ requires like the larger torque/power exertion of hip flexors and extensors $(11,12)$.

From a mechanical perspective, any motion ( $\boldsymbol{\alpha}$ :acceleration, $\boldsymbol{\alpha}$ :angular acceleration, $)$ is determined by the combination of force-related $(\boldsymbol{f}$ : forces, $\boldsymbol{\tau}$ : torques) and inertia-related ( $m$ : mass, $\boldsymbol{I}$ : moment of inertia) factors in line with Newton-Euler equations of motion:

$$
\begin{aligned}
& m \boldsymbol{a}=\sum \boldsymbol{f}, \\
& \boldsymbol{I} \boldsymbol{\alpha}=\sum \boldsymbol{\tau} .
\end{aligned}
$$

As humans control their motion via joint torques induced by muscle forces, the relationship between the torque and moment of inertia (Eq. 2) is particularly important for human motor execution. The above muscular characteristics in sprinters (7-10) can be interpreted as an adaptation to force-related factors, but simultaneously, the morphological changes would involve changes in the mass distribution and thereby inertia-properties. As lean tissue density is larger than that of fat tissue $(13,14)$, the muscular hypertrophy would involve an increase in 
the lower-limb mass, leading to a general speculation of the trade-off between greater torque exertion ability and greater difficulty in moving the lower limb (i.e., moment of inertia). However, as discussed above, the muscle hypertrophy in sprinters (7-9) is inhomogeneous. This leads to our hypothesis that the mechanical difficulties in swinging the lower limb and the moment of inertia around the hip might not correspond to an increase in the lower-limb mass in sprinters. Anatomists have previously developed scaling coefficients to calculate body segment inertia parameters (BSIPs) for motion analysis. This is done using the direct measurements of elderly cadavers (15) and indirect measurements in vivo through medical images or surface scans such as gamma-ray scanning (16), computed tomography (17), magnetic resonance imaging $(\mathrm{MRI})(18,19)$, and three-dimensional laser scanners $(20)$. However, the effects of adaptation to motor experience on inertial properties have not been fully examined.

Based on the simple mechanical law shown in Eq. 2), an increase in the moment of inertia leads to ( I ) increasing musculoskeletal mechanical loads if the motion is consistent, and (II) decreasing angular acceleration if the joint torque exertions are consistent. Understanding the morphological adaptation from an inertial perspective would provide useful knowledge for human motor performance development from both the injury prevention and performance improvement perspectives. In this study, we examined the inertia properties of sprinters by analyzing water- and fat-separated MRIs. Among the inertial properties, we focused on the normalized lower-limb moment of inertia around the hip joint $\left(\hat{I}_{\text {LowerLimb }}^{\text {Hip }}\right)$ because of the high musculoskeletal demands in the lower limb swing during sprinting (11, 12).

\section{Results}

The body size parameters in sprinters were significantly larger than those in nonsprinters (all $p<0.01, d=1.60,2.16$; Table $S 1$ ). The absolute values of the lower-limb mass and moment of inertia were significantly correlated to the body size parameters, while the relative mass and normalized moment of inertia were not (Fig. S1).

The relative mass of the entire lower limb was larger in sprinters $(18.7 \pm 0.7 \%$ body mass) than in non-sprinters $(17.6 \pm 0.6 \%$ body mass $)(p<0.01, d=1.73$; Fig. $1 \mathrm{~A})$. However, 
$\hat{I}_{\text {LowerLimb }}^{\text {Hip }}$ in sprinters $(0.044 \pm 0.002$ [a. u.]) and non-sprinters $(0.042 \pm 0.002$ [a. u.]) did not differ significantly ( $p=0.15, d=0.62$; Fig. 1B). From individual plots, we can observe that, although one of the non-sprinters showed a small $\hat{I}_{\text {LowerLimb }}^{\text {Hip }}$ (Fig. 1B), the $\hat{I}_{\text {LowerLimb }}^{\text {Hip }}$ in nonsprinters and sprinters, except for him, were highly comparable.

The relative thigh mass was significantly larger in sprinters $(12.9 \pm 0.4 \%)$ than in nonsprinters $(11.9 \pm 0.5 \%)(p<0.01, d=2.01)$, while those in the shank $(4.5 \pm 0.3 \%$ vs $4.3 \pm 0.3 \%)$ and foot $(1.4 \pm 0.1 \%$ vs $1.4 \pm 0.1 \%)$ were similar between them $(p=0.13,0.61, d=0.67,0.22$; Fig. $2 A)$. Compared to non-sprinters, all lower-limb segments in sprinters had a significantly larger lean mass ratio (thigh: $81.7 \pm 2.0 \%$ vs $72.0 \pm 4.8 \%$; shank: $73.0 \pm 2.0 \%$ vs $66.9 \pm 3.1 \%$; foot: $52.3 \pm 2.5 \%$ vs $48.3 \pm 1.9 \%$; all $p<0.01, d=1.85-2.62$ ) and smaller fat mass ratio (thigh: $10.3 \pm 2.1 \%$ vs $19.2 \pm 5.6 \%$; shank: $10.2 \pm 2.7 \%$ vs $15.3 \pm 3.5 \%$; foot: $19.6 \pm 1.6 \%$ vs $24.0 \pm 2.5 \%$; all $p<0.01$, $d=1.64-2.09$ ) (Fig. 2B). The mass ratio of the bone tissue in the thigh was smaller in sprinters $(8.1 \pm 0.6 \%)$ than in non-sprinters $(8.9 \pm 1.1 \%)(p=0.03, d=1.00$; Fig. $2 C)$, while those in the shank and foot were similar between the groups $(p=0.24,0.72, d=0.51$, and 0.15 ; Fig. 2B). The densities of all lower-limb segments were significantly larger in sprinters than in nonsprinters (thigh: $1.064 \pm 0.004 \mathrm{~g} / \mathrm{cm}^{3}$ vs $1.049 \pm 0.012 \mathrm{~g} / \mathrm{cm}^{3}$; shank: $1.084 \pm 0.007 \mathrm{~g} / \mathrm{cm}^{3}$ vs $1.073 \pm 0.008 \mathrm{~g} / \mathrm{cm}^{3}$; foot: $1.061 \pm 0.003 \mathrm{~g} / \mathrm{cm}^{3}$ vs. $\left.1.051 \pm 0.008 \mathrm{~g} / \mathrm{cm}^{3}\right)($ all $p<0.01, d=1.29$ 1.60; Fig. 2C). We showed the complete scaling coefficients to calculate the lower-limb segment inertia parameters for sprinters and non-sprinters in Supplemental Table S2. The radii of gyration, the moment of inertia normalized by the segment length, and segment mass around the segmental mediolateral axis in the thigh $(27.7 \pm 0.3 \%$ vs $27.9 \pm 0.2 \% ; p=0.02$, $d=1.08)$ and shank ( $27.0 \pm 0.3 \%$ vs. $27.6 \pm 0.3 \% ; p<0.01, d=2.24)$ were significantly smaller in sprinters than in non-sprinters.

For the values relating to the examiner's manual processing (digitizing of the anatomical landmarks on MRIs), we confirmed the excellent repeatability in segment lengths with the intraclass correlation coefficients (ICCs) and coefficient of variation (CVs) of 0.993 and $0.01-0.36 \%$ for the thigh, 0.994 and $0.00-0.57 \%$ for the shank, and 0.963 and $0.17-$ $1.44 \%$ for the foot.

\section{Discussion}


We found that sprinters have lower limbs with a larger relative mass but not a larger $\hat{I}_{\text {LowerLimb }}^{\text {Hip }}$ than non-sprinters. Although muscular adaptation, as previously demonstrated (79), increases the force and power exertion capacity, the increase in mass due to hypertrophy has been speculated to make it difficult for the body to move in terms of inertia. However, we revealed that the mechanical difficulty in swinging the lower limb in sprinters does not increase relatively.

A notable difference in the segment mass between sprinters and non-sprinters was confirmed only in the thigh. Moment of inertia $I$ is mechanically proportional to the square of the radius of gyration $r$ :

$$
I=m r^{2} .
$$

For example, if same mass (e.g., $1 \mathrm{~kg}$ ) is added to the thigh and shank in the sprinters in this study $\left(r_{\text {Hip-to-ThighCoM }}: 0.19 \mathrm{~m} ; r_{\text {Hip-to-ShankCoM }}: 0.60 \mathrm{~m}\right.$, Table S1), the increase in lower-limb moment of inertia due to the added mass in the thigh $\left(0.036 \mathrm{~kg} \cdot \mathrm{m}^{2}\right)$ is only $1 / 10$ of that due to the added mass to the shank $\left(0.360 \mathrm{~kg} \cdot \mathrm{m}^{2}\right)$. Thus, bottom-heavy, top-light feature of the lower limb in sprinters can be an optimal solution for their performance, from the biomechanical perspective. All the thigh, shank, and foot segments in sprinters had greater lean tissue ratios, smaller fat tissue ratios, and greater densities than those in non-sprinters. The sprinter/nonsprinters ratios of segment density were very similar in all the three segments, including the thigh (approximately 101\%). The sprinter/non-sprinters ratio of thigh density was smaller than that of the thigh mass $(107.6 \%)$. These results indicate that the segment mass is more affected by the segment volume than the segment density. Furthermore, the bone mass ratio of the sprinters was nearly $10 \%$ smaller than that of non-sprinters only in the thigh, which is straightforward to interpret as the adaptation to sprinting involves an increase in segment volume only in the thigh. Taken together, we indicated that the lower-limb inertia properties in sprinters, having a larger relative mass but not a larger $\hat{I}_{\text {LowerLimb }}^{\text {Hip }}$, are the result of the hypertrophic potential being greater only in the thigh.

The proximal-segment-specific hypertrophic potential in the lower limb in sprinters is related to the muscle-tendon arrangement. The length ratio of the tendon to the muscle in a muscle-tendon unit (MTU) in humans has a large variation (0.01-11.25), which is generally because proximal MTUs have shorter tendons compared to distal ones (21). Kubo et al. (22) 
showed that the Achilles tendon thickness in sprinters and non-sprinters was similar (sprinters/non-sprinters: $98.6 \%$ ), whereas the ankle plantar flexor muscle thicknesses were larger in sprinters than in non-sprinters (113.8-116.8\%). A previous meta-analysis (23) also showed a slight effect (weighted average effect size: 0.24 ) on the tendon hypertrophy in response to various types of exercise interventions. As such, the tendon tissue is hardly hypertrophic. Because of the difference in the hypertrophic potential between the muscle and tendon, the distal segment with a larger tendon length ratio results in a smaller volumetric (and mass) plasticity in the distal segment. Thus, it is suggested that the inter-segment differences in the hypertrophy are not a unique feature of sprinters, but the result of an adaptation potential in general in humans to explosive physical exercise. Regarding the significance of a larger tendon length ratio in the distal segment, a simulation study (24) reported that a larger mechanical energy generation is obtained with a longer tendon when the mass is small and vice versa. This suggests the morphological advantage of the muscletendon arrangement in humans for executing explosive motion from the force/work exertion perspective. However, to the best of our knowledge, the morphological advantages for the inertial properties in humans have not been examined. Based on simple mechanical laws, a small inertia can lead to both decreasing musculoskeletal demands and increasing body segment acceleration. The lower limb inertia properties found in sprinters, having a larger mass (related to muscle potential) but not a larger $\hat{I}_{\text {LowerLimb }}^{\text {Hip }}$, are beneficial both in preventing injury and improving performance. As discussed above, the increase in the lower-limb moment of inertia due to the added mass to the shank is 10 -fold of that due to the added mass to the thigh; a smaller volumetric (and mass) plasticity in the distal segment is an important factor to keep the lower-limb moment of inertia small. Thus, our findings further add quantified evidence showing a novel significant advantage of the muscle-tendon arrangement for the adaptability to explosive (sprinting) motion from an inertial perspective.

We found differences in the normalized body segment inertia parameters (BSIPs), such as the radii of gyration, between sprinters and non-sprinters. Researchers have previously developed scaling coefficients to calculate BSIPs for motion analysis using direct measurements of elderly cadavers (15) and in vivo medical imaging such as gamma-ray scanning (16), computed tomography $(17)$, or $\operatorname{MRI}(18,19)$; however, these scaling factors do 
not adequately accommodate the effects of morphological adaptation on the inertial properties in athletes. We showed the complete scaling coefficients to estimate the BSIPs of the sprinter's lower-limb segments as supplemental data, which provides sufficient scaling equations to calculate the hip, knee, and ankle joint dynamics. Although it is slightly outside the scope of this study, our data allow future studies to analyze motion in male sprinters more accurately.

Below, we discuss the methodological advantages and limitations of the present study. First, most of our MRI segmentation processes were computationally and automatically performed by the widely used standard Otsu's binarization algorithm (25) on fat/waterseparated images, leading to advantages in the methodological simplicity and robust results. Alternately, the landmark position coordinates were acquired by manual digitizing on the MRIs, having potential effects on the segment length used for data normalization. However, we acquired MRI images with a slice thickness of $2 \mathrm{~mm}$, which is thinner than the previous studies examining inertia properties [for example, Sreenivasa et al. (26): $6 \mathrm{~mm}-48 \mathrm{~mm}$; Pearsall et al. (27): $10 \mathrm{~mm}$; Cheng et al. (18): $20 \mathrm{~mm}$ ]. We confirmed excellent repeatability of the segment lengths (ICC $>0.96$ and $\mathrm{CV}<1.5 \%)$; thus, the potential effect of manual digitizing on the segment length would not critically alter our results. Second, non-sprinters had similar body sizes as the average Japanese 20 year old males shown in the 2019 Japanese Government statistics (28), while the sprinters were larger than the standard Japanese population, which might affect our conclusion. However, we assessed the dimensionless inertia parameters calculated according to Hof (29) to eliminate the effect of body size. Although there were significant correlations between the absolute inertia and body size parameters, the dimensionless values (the relative mass and the normalized moment of inertia of the whole lower limb around the hip joint) did not correlate with the body size, suggesting that these values could reflect the individual differences in body shapes and mass distributions, thereby not being critically affected by the inter-group differences in the body size. Third, although we determined the sample size of each group as 12 based on a priori power analysis (see Methods section), we excluded one of the sprinter's data due to an error in the water/fat separation of his foot scan. However, the main findings regarding intergroup differences were statistically significant, which suggest that the exclusion did not critically 
affect our conclusion.

We examined only male participants. Generally, body composition has large sex differences (30); therefore, our findings might not be directly applicable to females, which is an important future theme. It is also unclear whether our findings are applicable to the upper limbs. It has been suggested that high-speed throwing in the upper limbs is one of the factors for evolution in current humans (31). It would not be surprising if humans also have morphological advantages in their upper limbs from an inertial perspective. Furthermore, unlike the lower limbs, which constantly support the body against gravity, the upper limbs, being free from such constant gravitational load, would reflect pure trainability in humans; the morphological adaptation in the upper limbs is an interesting future theme for understanding human plasticity in motor performance. Nevertheless, our findings regarding noncorrespondence with an increase in lower-limb mass and moment of inertia in male sprinters open a novel perspective, inertia, for understanding human plasticity.

In conclusion, we demonstrate the lower-limb morphology in sprinters with a larger relative mass but with a not larger normalized moment of inertia around the hip comparing it to non-sprinters. This result suggests one of the practical advantages of human morphological characteristics-a larger tendon length ratio in the distal segment muscles-for their adaptation to explosive motor tasks, which provides a novel perspective to the literature for understanding human morphological adaptability.

\section{Materials and Methods}

\section{Experimental design}

We performed a priori power analysis for an independent $t$-test with the parameters of type I error; $\alpha=0.05$, statistical power: $1-\beta=0.8$, and effect size: Cohen's $d=1.2$ 'very large' (32), which calculated the total sample size ( $n)$ as 24 ( $n=12$ in each group). According to the power analysis, we recruited a total of 24 healthy males, including 12 well-trained (athletic career $>7$ years and $100 \mathrm{~m}$ best records: $10.44-10.83 \mathrm{~s}$ ) sprinters and 12 non-sprinters who did not undertake resistance training or sports activities for at least 2 years. However, the data of one of the sprinters could not be analyzed because of an MRI error (fat/water separation error in the Dixon sequence) around the endpoint of the toe in the foot segment scan, and the 
main outcome data (i.e., the lower-limb mass and the moment of inertia around the hip joint) could not be calculated. Thus, we excluded this participant's data from the analysis. The analyzed participants were 11 sprinters (age: $20 \pm 2$ yrs, height: $1.77 \pm 0.06 \mathrm{~m}$; body mass: $68.2 \pm 4.4 \mathrm{~kg}$, Mean $\pm \mathrm{SD}$ ) and 12 non-sprinters (age: $23 \pm 3 \mathrm{yrs}$, height: $1.69 \pm 0.04 \mathrm{~m}$; body mass: $58.6 \pm 4.5 \mathrm{~kg}$ ). The purpose and experimental protocol of the study were explained to the participants and they provided written informed consent. The Human Research Ethics Committee at Waseda University, Japan approved the study protocol (reference number: 2019-174).

We used a 3.0 Tesla MR scanner (SIGNA Premier, GE Healthcare, Milwaukee, USA) to obtain the MR scans. All lower limb segment scans were performed in the supine position. The 2-point 3D-dixon axial sequence, called "Lava-Flex" in GE Healthcare, was performed on the right thigh, shank, and foot segments ('Spacing-Between-Slices': $2 \mathrm{~mm}$, 'Pixel-Spacing': [0.2930 0.2930] mm, Width $\times$ Height: $1024 \times 1024$ pixels, other detail information can be confirmed in the Supplemental material). Each scan of the thigh and shank had two sections because of the limitation in the length scanned in one section. The two sections of each thigh and the shank scans were taken continuously without changing the position on the MRI machine. In the MRI settings, the anteroposterior and mediolateral coordinates of the two consecutive scans coincided. To minimize the effect of magnetic field inhomogeneity on the Dixon sequence, the superoinferior ranges of the two scans overlapped by $>10 \mathrm{~cm}$.

\section{MRI processing}

We analyzed the 16-bit depth MRI images using MATLAB 2019a (MathWorks, Natick, MA, USA). A flowchart of the segmentation procedure is presented in Fig. S2. To create a temporal body mask, we first created fat-only and water-only temporal binary images using a low intensity threshold. These binary images were added together, and the temporal body mask was defined by filling any internal holes in the added masks based on a binary morphology operation (33). After creating the temporal body mask, we removed the pixel intensity in the unwanted areas (other than the range of interest, such as the part of the opposite leg segment included in the image). After that, we estimated and then homogenized the inhomogeneity field using a grayscale morphological closing operation with a large 
circular structure element (33). The threshold for the foreground and background signal intensity of each of the fat-only and water-only images was determined using Otsu's method (25), which created water-binary and fat-binary images. We created a definitive body mask by adding the two masks together and filling any internal holes with a binary morphology operation (33). We classified each pixel as a fat tissue, lean (muscle or skin) tissue, or a background using water- and fat-binary images. For a slice having some voxels involved in both water- and fat-binary images, we further performed $k$-means clustering to classify the intensity of the voxels in the sum of the fat-only and water-only images (i.e., in-phase image) into three categories (fat tissue, lean tissue, or background), which were applied to the voxels involved in both the water-binary and fat-binary images.

The trabecular bone has MRI intensity characteristics similar to fat tissue because of the yellow marrow (26), while the cortical bone is not brightened by MRI. These bone tissues were classified as follows (Fig. S2):

Step 1. Search for a region inside the body mask region where the pixels classified as fat tissue (trabecular bone in fact) are surrounded by pixels classified as background (cortical bone in fact) and define them as trabecular bone and cortical bone.

Step 2. (When a bone cannot be explored in Step 1 and the trabecular bone is an independent object in the fat-binary image), select and define the independent object in the fat-only image as a trabecular bone.

Step 3. (When a trabecular bone was in contact with the outer fat tissue in the fatbinary image and could not be defined in Steps 1 and 2) Manually draw a border with the fat in the fat-only image or in-phase image using a pen tablet (Artist 15.6 Pro, XP-PEN, Japan) and define the object as a trabecular bone.

Step 4. (After Steps 2 and 3) Define the pixels that surround the trabecular bone and are classified as background (cortical bone in fact) as a cortical bone.

Step 5. Adjust the cortical bone if necessary (for example, if the tissue adjacent to the cortical bone that is not depressed on MRI is considered to be a cortical bone).

\section{Data calculation}


The 3D coordinates of the anatomical landmarks (Table S3) on the MR coordinate system were derived via manual digitizing in the 3D mode using the OsiriX software version 13 (Pixmeo, Geneva, Switzerland) by an examiner. The examiner repeated the digitization of all landmarks from eight participants for whom it had been $>1$ month following the first digitizing process to assess the intra-examiner repeatability of manual digitizing.

The ankle joint in the foot and shank scans and the knee joint in the shank and thigh scans were defined as the midpoints between the malleoli and the lateral and medial articular cleft of the knee, respectively. The hip joint was defined using a least-squares calculation for a sphere fitting of 30 points distributed over the surface of the femoral head, similar to Harrington et al. (34). The ankle and knee boundary planes were defined as the horizontal planes passing those joint locations (Fig. 3A). The hip joint boundary was defined by a plane $37^{\circ}(35,36)$ vertical on the medial to the hip joint and a horizontal plane lateral to the hip joint (27) (Fig. 3A). The lean and fat voxels outside of the border were excluded. The cortical and trabecular bone voxel in that segment was retained even though it was outside the proximal and distal borders. Bone voxels within the segment were retained even if they were outside the boundary, whereas bone voxels in another segment were excluded even if they were inside the boundary.

Based on the literature values $(13,14)$, we defined the tissue density as:

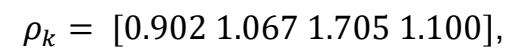

where $k=1,2,3,4$ shows the tissues of fat, lean, cortical bone, and trabecular bone, respectively. The mass, $m_{s}$, volume $V_{s}$, density $\rho_{s}$, and position coordinates of the center of mass, $\left[\begin{array}{l}x_{s, \mathrm{CoM}} \\ y_{s, \mathrm{CoM}} \\ z_{s, \mathrm{COM}}\end{array}\right]$, of a segment $s$ were calculated as

$$
\begin{gathered}
m_{s}=\sum_{k=1}^{4} \sum_{i=1}^{n_{k}}\left[\left(w^{2} h\right) \rho_{k}\right] \\
V_{s}=\sum_{k=1}^{4}\left[\left(w^{2} h\right) n_{k}\right] \\
\rho_{s}=\frac{m_{s}}{V_{s}}
\end{gathered}
$$




$$
\left[\begin{array}{l}
x_{s, \mathrm{CoM}} \\
y_{s, \mathrm{CoM}} \\
z_{s, \mathrm{CoM}}
\end{array}\right]=\frac{1}{m_{s}} \sum_{k=1}^{4} \sum_{i=1}^{n_{k}}\left[\left(w^{2} h\right) \rho_{k}\left[\begin{array}{l}
x_{i} \\
y_{i} \\
z_{i}
\end{array}\right]\right]
$$

where $n_{k}$ is the number of voxels classified as the tissue $k, w$ and $h$ were the width ('PixelSpacing': $0.2930 \mathrm{~mm}$ ) and height ('Spacing-Between-Slices': $2.0000 \mathrm{~mm}$ ) of each voxel (i.e., $w^{2} h$ is the volume of each voxel), and $\left[\begin{array}{l}x_{i} \\ y_{i} \\ z_{i}\end{array}\right]$ is the position coordinate of the $i$ th voxel, respectively.

Inertia tensor of segment $s, I_{s}^{\mathrm{GCS}}$, was calculated as shown below:

$$
\boldsymbol{I}_{s}^{\mathbf{G C S}}=\left[\begin{array}{ccc}
\sum_{i=1}^{n}\left[I_{x_{i}}+m_{i}\left(y_{i}{ }^{2}+z_{i}{ }^{2}\right)\right] & -\sum_{i=1}^{n}\left(m_{i} x_{i} y_{i}\right) & -\sum_{i=1}^{n}\left(m_{i} z_{i} x_{i}\right) \\
-\sum_{i=1}^{n}\left(m_{i} x_{i} y_{i}\right) & \sum_{i=1}^{n}\left[I_{y_{i}}+m_{i}\left(z_{i}{ }^{2}+x_{i}{ }^{2}\right)\right] & -\sum_{i=1}^{n}\left(m_{i} y_{i} z_{i}\right) \\
-\sum_{i=1}^{n}\left(m_{i} z_{i} x_{i}\right) & -\sum_{i=1}^{n}\left(m_{i} y_{i} z_{i}\right) & \sum_{i=1}^{n}\left[I_{z_{i}}+m_{i}\left(x_{i}{ }^{2}+y_{i}{ }^{2}\right)\right]
\end{array}\right]
$$

where $m_{i}$ is the mass of $i$ th voxel, $I_{x_{i}} I_{y_{i}}$ and $I_{z_{i}}$ were the moments of inertia of each voxel and were calculated as follows.

$$
I_{x_{i}}=I_{y_{i}}=\frac{m_{i}\left(w^{2}+h^{2}\right)}{12} \text { and } I_{z_{i}}=\frac{m_{i}\left(2 w^{2}\right)}{12}
$$

A right-handed orthogonal segment coordinate system (SCS) was fixed at each of the thigh, shank, and foot segments using the 3D coordinates of landmarks. This definition was consistent with that used by Sado et al. $(37,38)$. We calculated the inertia tensor in each $\operatorname{SCS}\left(I_{s}^{\text {SCS }}\right)$ as follows:

$$
I_{S}^{S C S}=R_{s}{ }^{\prime} I_{S}^{\mathrm{GCS}} R_{S}
$$

where $\boldsymbol{R}_{\boldsymbol{s}}$ is the rotational transformation matrix from the SCS to the GCS of segment $s$. Note that the principal axes of the segments are not consistent with the axes of the SCSs (39); thus, $I_{s}^{\text {SCS }}$ is not in the form of a diagonal matrix, which is similar to Dumas et al. (39), considering the differences between the SCS axes and the inertial principal axes.

We hypothetically created the lower-limb data in an anatomical position from the 3D coordinates in the MRI coordinate system of the thigh, shank, and foot using hierarchically constructed coordinates with the hip as the origin (Fig. 3B). In this hypothetical creation, the $\boldsymbol{x}$-axis directions of their SCSs were consistent with each other. We then calculated $I_{\text {LowerLimb }}^{\text {Hip }}$ 
in the data of this hypothetical anatomical position as

$$
I_{\text {Lowerlimb }}^{\text {Hip }}=\sum_{s=1}^{3}\left\{I_{s}^{\text {SCS }}{ }_{x x}+m_{s}\left(\left|y_{s}-y_{\text {Hip }}\right|^{2}+\left|z_{s}-z_{\text {Hip }}\right|^{2}\right)\right\}
$$

where $I_{s}^{S C S}{ }_{x x}$ is the moment of inertia of the segment around its CoM, $y_{s}, y_{\mathrm{Hip}}, z_{s}$ and $z_{\mathrm{Hip}}$ are the anteroposterior or superoinferior coordinates of the CoM of segment $s$ or the hip joint in a hypothetically created coordinate. In this coordinate system, the origin is set at the hip joint; thus, $y_{\text {Hip }}$, and $z_{\text {Hip }}$ are equal to zero.

The segment and the lower-limb masses were expressed relative to the whole-body mass. Both the products and the moments of inertia of each segment were expressed relative to the segment length $l_{s}$ and the segment mass $m_{s}$. For example, the moment of inertia of segment $s$ around the $\boldsymbol{x}$-axis is

$$
r_{s x x}=\frac{\sqrt{\frac{I_{s}^{\text {SCS }} m_{s x}}{m_{s}}}}{l_{s}}
$$

The segment lengths were defined as the Euclidean distances between the proximal and distal joint locations (Fig. 3B). Using the scaling method of gait data established by Hof (29), we normalized $I_{\text {LowerLimb }}^{\text {Hip }}$ by the lower-limb length $\left(l_{\text {Lowerlimb }}\right)$ and the whole-body mass $\left(m_{\text {body }}\right)$ as:

$$
\hat{I}_{\text {LowerLimb }}^{\text {Hip }}=\frac{I_{\text {LowerLimb }}^{\text {Hip }}}{m_{\text {body }} l_{\text {Lowerlimb }}^{2}}
$$

where $l_{\text {Lowerlimb }}$ is the height difference between the hip joint center and calcaneus in the hypothetically created coordinates (Fig. 3B).

\section{Statistical analysis}

We used MATLAB 2019a for statistical analysis. We used ICCs and CVs to assess the repeatability of the examiners' manual-digitising-basis segment lengths. The ICCs can be interpreted as good $(>0.75)$ or excellent $(>0.90)$ (37). After normality confirmation using the Jarque-Bera test, we calculated the Pearson's correlation coefficient $(r)$ to confirm the effect of body size on the lower-limb inertia properties. We tested the significance of the intergroup differences using an independent $t$-test. The statistical significance was set at 0.05 . The effect size of each $t$-test was determined using Cohen's $d(40)$ with classification as small $(\leq 0.49)$, 
medium (0.50-0.79), large (0.80-1.19), very large (1.20-1.99), and huge $(\geq 2.00)(32)$.

\section{Acknowledgements}

We would like to thank Dr. Gaku Kakehata for his help in participant recruitment.

\section{References}

1. N. M. Young, G. P. Wagner, B. Hallgrímsson, Development and the evolvability of human limbs. Proc. Natl. Acad. Sci. U. S. A. 107, 3400-5 (2010).

2. D. M. Bramble, D. E. Lieberman, Endurance running and the evolution of Homo. Nature 432, 345-352 (2004).

3. Japan Sports Agency, The Report of FY 2020 Survey on Physical Strength and Athletic Performance (2021).

4. T. Fukunaga, et al., Muscle volume is a major determinant of joint torque in humans. Acta Physiol. Scand. 172, 249-255 (2001).

5. T. D. O'Brien, N. D. Reeves, V. Baltzopoulos, D. A. Jones, C. N. Maganaris, Strong relationships exist between muscle volume, joint power and whole-body external mechanical power in adults and children. Exp. Physiol. 94, 731-738 (2009).

6. T. G. Balshaw, T. M. Maden-Wilkinson, G. J. Massey, J. P. Folland, The Human Muscle Size and Strength Relationship: Effects of Architecture, Muscle Force, and Measurement Location. Med. Sci. Sport. Exerc. Publish Ah (2021).

7. R. Ema, M. Sakaguchi, Y. Kawakami, Thigh and Psoas Major Muscularity and Its Relation to Running Mechanics in Sprinters. Med. Sci. Sport. Exerc. 50, 2085-2091 (2018).

8. N. Tottori, et al., Trunk and lower limb muscularity in sprinters: what are the specific muscles for superior sprint performance? BMC Res. Notes 14, 10-15 (2021).

9. R. Miller, et al., The Muscle Morphology of Elite Sprint Running. Med. Sci. Sport. Exerc. 53, 804-815 (2020).

10. K. Takahashi, K. Kamibayashi, T. Wakahara, Muscle size of individual hip extensors in sprint runners: Its relation to spatiotemporal variables and sprint velocity during maximal velocity sprinting. PLoS One 16, 1-12 (2021). 
11. A. G. Schache, et al., Effect of running speed on lower limb joint kinetics. Med. Sci. Sports Exerc. 43, 1260-1271 (2011).

12. T. W. Dorn, A. G. Schache, M. G. Pandy, Muscular strategy shift in human running: dependence of running speed on hip and ankle muscle performance. J. Exp. Biol. 215, 1944-56 (2012).

13. H. K. Huang, S. C. Wu, The evaluation of mass densities of the human body in vivi from CT Scans. Comput. Biol. Med. 6, 337-343 (1976).

14. P. E. Martin, M. Mungiole, M. W. Maezke, J. M. Longhill, The use of magnetic resonance imaging for measuring segment inertial propertierties. J. Biomech. 22, 367376 (1989).

15. C. E. Clauser, J. T. McConville, J. W. Young, Weight, Volume, and Center of Mass of Segments of the Human Body. Natl. Tech. Inf. Serv., 1-112 (1969).

16. P. de Leva, Adjustments to Zatsiorsky-Seluyanov's segment inertia parameters. J. Biomech. 29, 1223-1230 (1996).

17. H. K. Huang, F. R. Suarez, Evaluation of cross-sectional geometry and mass density distributions of humans and laboratory animals using computerized tomography. $J$. Biomech. 16, 821-832 (1983).

18. C. K. Cheng, H. H. Chen, C. S. Chen, C. L. Lee, C. Y. Chen, Segment inertial properties of Chinese adults determined from magnetic resonance imaging. Clin. Biomech. 15, 559-566 (2000).

19. M. Mungiole, P. E. Martin, Estimating segment inertial properties: Comparison of magnetic resonance imaging with existing methods. J. Biomech. 23, 1039-1046 (1990).

20. G. Shan, C. Bohn, Anthropometrical data and coefficients of regression related to gender and race. Appl. Ergon. 34, 327-337 (2003).

21. M. G. Hoy, F. E. Zajac, M. E. Gordon, A musculoskeletal model of the human lower extremity: The effect of muscle, tendon, and moment arm on the moment-angle relationship of musculotendon actuators at the hip, knee, and ankle. J. Biomech. 23, 157-169 (1990).

22. K. Kubo, T. Ikebukuro, H. Yata, M. Tomita, M. Okada, Morphological and mechanical 
properties of muscle and tendon in highly trained sprinters. J. Appl. Biomech. 27, 336$344(2011)$.

23. S. Bohm, F. Mersmann, A. Arampatzis, Human tendon adaptation in response to mechanical loading: a systematic review and meta-analysis of exercise intervention studies on healthy adults. Sport. Med. - Open 1 (2015).

24. A. Nagano, T. Komura, S. Fukashiro, Effects of the length ratio between the contractile element and the series elastic element on an explosive muscular performance. $J$.

Electromyogr. Kinesiol. 14, 197-203 (2004).

25. N. Otsu, A threshold selection method from gray-level histograms. IEEE Trans. Syst. Man Cybern. 9, 62-66 (1996).

26. M. Sreenivasa, C. J. G. Chamorro, D. Gonzalez-Alvarado, O. Rettig, S. I. Wolf, Patient-specific bone geometry and segment inertia from MRI images for model-based analysis of pathological gait. J. Biomech. 49, 1918-1925 (2016).

27. D. J. Pearsall, J. G. Reid, R. Ross, Inertial properties of the human trunk of males determined from magnetic resonance imaging. Ann. Biomed. Eng. 22, 692-706 (1994).

28. Government of Japan, e-Stat: Portal Site of Official Statistics of Japan (2020).

29. A. L. Hof, Scaling gait data to body size. Gait Posture 4, 222-223 (1996).

30. M. A. Bredella, "Sex Differences in Body Composition BT - Sex and Gender Factors Affecting Metabolic Homeostasis, Diabetes and Obesity" in F. Mauvais-Jarvis, Ed. (Springer International Publishing, 2017), pp. 9-27.

31. N. T. Roach, M. Venkadesan, M. J. Rainbow, D. E. Lieberman, Elastic energy storage in the shoulder and the evolution of high-speed throwing in Homo. Nature 498, 483-6 (2013).

32. S. S. Sawilowsky, New Effect Size Rules of Thumb. J. Mod. Appl. Stat. Methods 8, 597-599 (2009).

33. J. D. Klingensmith, A. L. Elliott, M. Fernandez-del-Valle, S. Mitra, Automated segmentation of cardiac adipose tissue in Dixon magnetic resonance images. $\mathrm{J}$. Biomed. Graph. Comput. 8, 1 (2017).

34. M. E. Harrington, A. B. Zavatsky, S. E. M. Lawson, Z. Yuan, T. N. Theologis, 
Prediction of the hip joint centre in adults, children, and patients with cerebral palsy based on magnetic resonance imaging. J. Biomech. 40, 595-602 (2007).

35. M. Rossi, A. Lyttle, A. El-Sallam, N. Benjanuvatra, B. Blanksby, Body segment inertial parameters of elite swimmers using DXA and indirect methods. J. Sport. Sci. Med. 12, 761-775 (2013).

36. D. J. Pearsall, J. G. Reid, L. a Livingston, Segmental inertial parameters of the human trunk as determined from computed tomography. Ann. Biomed. Eng. 24, 198-210 (1996).

37. N. Sado, S. Yoshioka, S. Fukashiro, A non-orthogonal joint coordinate system for the calculation of anatomically practical joint torque power in three-dimensional hip joint motion. Int. J. Sport Heal. Sci. 15, 111-119 (2017).

38. N. Sado, S. Yoshioka, S. Fukashiro, Curved approach in high jump induces greater jumping height without greater joint kinetic exertions than straight approach. Med. Sci. Sport. Exerc. 54, 120-128 (2022).

39. R. Dumas, L. Chèze, J. P. Verriest, Adjustments to McConville et al. and Young et al. body segment inertial parameters. J. Biomech. 40, 543-553 (2007).

40. J. Cohen, Statistical power analysis for the behavioral sciences, 2nd Ed. (Lawrence Earlbaum Associates, 1988) https:/doi.org/10.1234/12345678. 


\section{Figures}

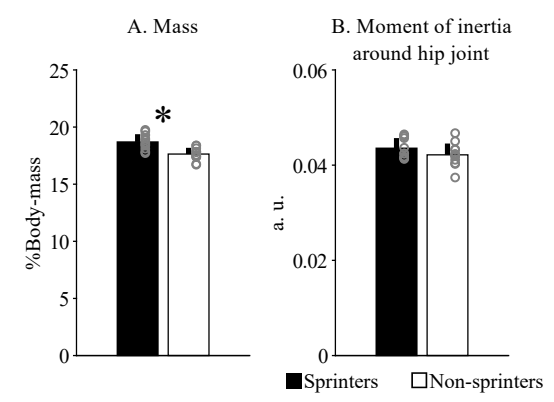

Fig. 1 Whole lower-limb inertia properties. Relative mass (A) and normalized

moment of inertia around the hip joint in hypothetically constructed anatomical position data (B).

Note: Bar graphs show means and standard deviations. Gray plots show individual data. Asterisk $\left(^{*}\right)$ indicates a significant difference between sprinters and nonsprinters. 

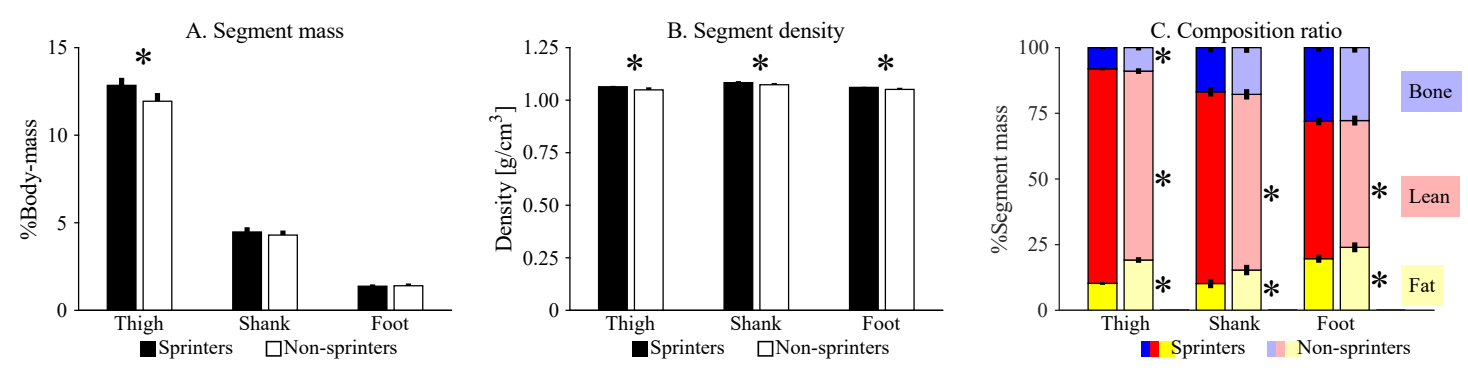

Fig. 2 Segment mass and its determinants of each lower-limb segment. Relative mass

(A), density (B), and composition ratio (C) in each of the thigh, shank, and foot segments.

Note: Bar graphs show mean and standard deviation. Asterisk ( $\left.{ }^{*}\right)$ indicates a significant difference between sprinters and non-sprinters. 
A
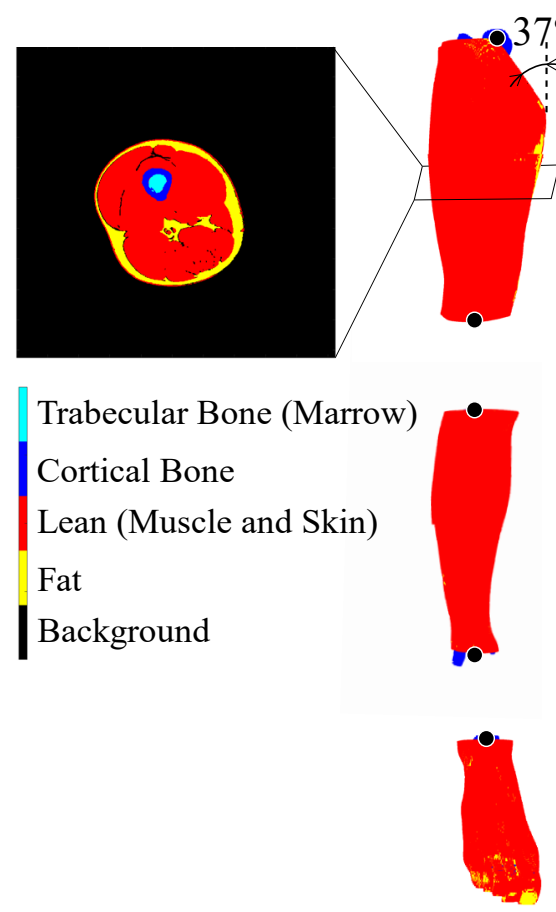

B

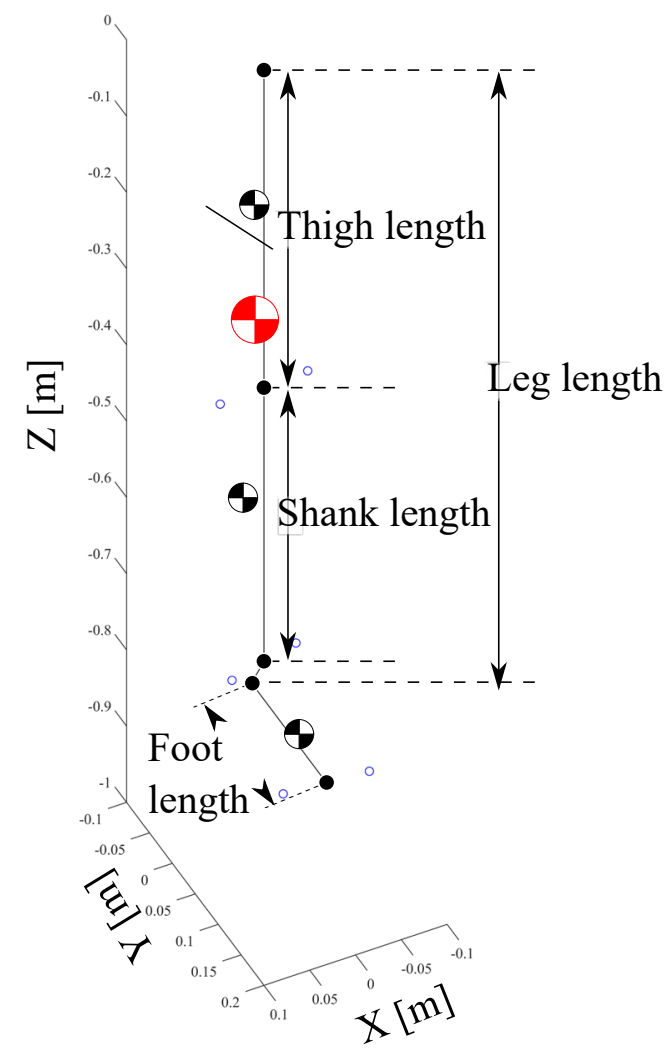

Fig. 3 A typical example of the constructed data. A three-dimensional constructed volume data of the thigh, shank and foot $(A)$ and a stick picture created from three-dimensional position coordinates of the entire hypothetically constructed lower limb (B).

Note: The origin of the hypothetical coordinates was at the hip joint center. The lower limb length was defined as the height difference between the hypothetical coordinates of the hip and calcaneus. 